\title{
Estabilidad semántica en la producción de atributos semánticos entre adultos mayores y adultos jóvenes
}

\section{Semantic Stability in the Production of Semantic Features between Senior and Young fidults \\ Estabilidade semântica na produção de atributos semânticos entre adultos idosos e adultos jouens}

\author{
Jorge Ricardo Vivas* \\ Sandra Martínez** \\ Deisy Krzemien ${ }^{* * *}$ \\ Francisco Lizarralde ${ }^{* * * *}$ \\ Universidad Nacional de Mar del Plata
}

Doi: https://doi.org/10.12804/revistas.urosario.edu.co/apl/a.7368

\section{Resumen}

En los últimos años, se ha producido una profusa investigación en torno a la memoria semántica en la vejez. En ese marco, el presente trabajo abordó la producción de atributos semánticos y las variaciones en la fluencia verbal en el envejecimiento cognitivo normal. Existen numerosos estudios sobre fluencia, pero son exiguos los que comparan la distancia semántica entre conceptos en poblaciones de personas jóvenes y mayores de una misma región. El objeto de este trabajo fue comparar y analizar la distancia semántica entre ambos grupos etarios y la riqueza

Dirigir correspondencia a Jorge Ricardo Vivas: Instituto de Psicología Básica, Aplicada y Tecnología, Universidad Nacional de Mar del Plata, Conicet; Facultad de Psicología, Universidad Nacional de Mar del Plata, Funes 3280, cuerpo V, nivel III -B7602AYJ, Mar del Plata, Argentina. Correo electrónico: jvivas@gmail.com

Para citar este artículo: Vivas, J., Matínz, S., Krzemien, D., \& Lizarralde, F. (2020). Estabilidad semántica en la producción de atributos semánticos entre adultos mayores y adultos jóvenes. Avances en Psicología Latinoamericana, 38(2), 1-19. https://doi. org/10.12804/revistas.urosario.edu.co/apl/a.7368 
semántica por medio de la fluencia dada a 130 conceptos por 90 adultos mayores de 65 años, cognitivamente conservados, con los atributos producidos por 120 adultos jóvenes, tal como se hallan en las Normas de Producción de Atributos Semánticos en Español. Estos atributos fueron empíricamente recolectados en forma escrita y corresponden a los dominios 'seres vivos' y 'no vivos'. Se encontraron diferencias significativas en fluencia verbal a favor de los más jóvenes, en coincidencia con lo expresado en la literatura, y estabilidad en la constitución semántica entre los conceptos dados por ambos grupos etarios, como aspecto novedoso.

Palabras clave: atributos; distancia semántica; fluencia verbal; adultos mayores; adultos jóvenes.

\section{fibstract}

In recent years, there has been a profuse research on semantic memory in old age. In this framework, this paper addressed the production of semantic features and the variations in verbal fluency in normal cognitive aging. There are numerous studies on fluency, but there are fewer that compare the semantic distance between concepts in populations of young people and seniors in the same region. The purpose of this paper is to compare and analyze the semantic distance between both age groups, and the semantic richness measured by the fluency, given to 130 concepts by 90 cognitively conserved adults over 65 years old, with the features produced by 120 young adults, as found in the Normas de Producción de Atributos Semánticos en Español. Semantic features were empirically collected in written form and correspond to the domains living and non-living beings. Significant differences were found in verbal fluency in favor of the youngest, in coincidence with what was expressed in the literature, and stability in the semantic constitution between the concepts given by both age groups, as a novel aspect.

Keywords: Features; semantic distance; verbal fluency; seniors; young adults.

\section{Resumo}

Nos últimos anos se tem produzido uma profunda pesquisa em torno ao tema da memória semântica na velhice. Nesse contexto, o presente trabalho abordou a produção de atributos semânticos e as variações na fluência verbal no envelhecimento cognitivo normal. Existem numerosos estudos sobre fluência, mas são exíguos os estudos que comparam a distância semântica entre conceitos em populações de jovens e de idosos de uma mesma região. O objetivo deste trabalho é comparar e analisar a distância semântica entre ambos os grupos etários e a riqueza semântica medida por meio da fluência, dada para 130 conceitos por 90 idosos de 65 anos cognitivamente conservados, com os atributos produzidos por 120 adultos jovens, tal como encontram-se nas Normas de Produção de Atributos Semânticos em Espanhol. Os atributos semânticos foram empiricamente coletados em forma escrita e correspondem aos domínios seres vivos e não vivos. Se encontraram diferenças significativas em fluência verbal a favor dos mais jovens, em coincidência com o expressado na literatura, e estabilidade na constituição semântica entre os conceitos dados por ambos os grupos etários, como aspeto inovador.

Palavras-chave: atributos; distância semântica; fluência verbal; idosos; adultos jovens.

Probablemente debido a su uso cotidiano y su disponibilidad directa para uso experimental, las palabras forman parte de los estímulos más utilizados en la psicología cognitiva, la neuropsicología, la neurociencia cognitiva, la psicolingüística y disciplinas vecinas. Este tipo de estímulos tiene una fuerte impronta cultural y, por lo tanto, posee unas características psicolingüísticas que varían idiosincráticamente de acuerdo al entorno sociocultural. Como consecuencia, se han realizado esfuerzos para obtener bases normativas de palabras en las que se ofrecen los valores correspondientes a ciertas características psicolingüísticas, con el fin de poder manipularlas y controlarlas experimentalmente. 
Dada la importancia de los atributos semánticos para las teorías sobre memoria semántica, se ha reconocido el valor de recolectar normas de producción de atributos para construir modelos, testear hipótesis, disponer de estímulos experimentales y generar tareas de evaluación en el ámbito clínico (McRae, Cree, Seidenberg \& McNorgan, 2005). Este tipo de normas se obtiene mediante la tarea de producción de atributos, en la cual se pide a un conjunto de participantes que indique características sobre un conjunto de conceptos. De esta forma, es posible disponer de un listado de los atributos semánticos que mejor definen dichos conceptos, con lo que se obtiene información que permite el cálculo de medidas relativas a estos y sus atributos, así como de la relación entre ellos. Esta tarea ha sido la principal estrategia utilizada por los investigadores del campo, aunque se ha empezado a señalar que, más allá de la robustez de la información generada, existe variabilidad intersubjetiva en sujetos del mismo colectivo lingüístico y, con más razón, en miembros de comunidades lingüísticas diversas (Chaigneau, Canessa, Barra \& Lagos, 2018).

Las normas permiten entender mejor la organización de la información semántica, las características y las relaciones entre atributos y su vinculación con las categorías semánticas (Vinson \& Vigliocco, 2008). El procesamiento semántico es un aspecto crucial; sin embargo, aún no cabalmente comprendido. Recientemente, Montefinese, Buchanan y Vinson (2018) desafiaron las explicaciones teóricas del priming automático por medio de tareas con conceptos concretos y abstractos. Ellos sugieren que el priming puede deberse a procesos estratégicos controlados de los participantes como la generación de expectativa prospectiva. El estudio de la similitud en la estructura de las representaciones semánticas parece requerir la elucidación del núcleo mismo del significado de los conceptos.

La teoría estándar de formación de conceptos (Ashby \& Alfonso-Reese, 1995) sostiene que los concretos se aprenden observando los ejemplos de categorías, abstrayendo los rasgos relevantes y estimando la distribución de frecuencias de esas propiedades relevantes (para una distinción con los conceptos abstractos véase Bolognesi Steen, 2019). Así, se afirma que las representaciones se basan en la percepción y se aprenden de acuerdo a los principios clásicos del aprendizaje. Por ejemplo, a través de la experiencia, las personas aprenden que un animal se caracteriza por poseer rasgos tales como tamaño, si posee pelos, tiene patas, nada, vuela, etc. Esta actividad le permite organizar una estructura semántica y realizar juicios de categoría como, por ejemplo, ¿qué tan típico es un pato de la categoría ave? ¿La ballena pertenece a la categoría pez o mamífero? ¿Cuán central es la propiedad ladra para la categoría perro?). La naturaleza de los procesos de elicitación y estimación de similitudes son la preocupación de muchas teorías que intentan explicar cómo las propiedades son combinadas para lograr la clasificación correcta.

Así, las teorías de los rasgos asumen que la información que proveen los atributos semánticos es una pieza primordial para la representación semántica (Baroni, Barbu, Murphy \& Poesio, 2010; Murphy, 2002; Riordan \& Jones, 2010; Smith, Shoben \& Rips, 1974; Taylor, Moss \& Tyler, 2007). Estos modelos se han focalizado en el estudio de variables que indican el nivel de conectividad entre un atributo y un concepto. Así, se propusieron la relevancia semántica (Sartori \& Lombardi, 2004), la distintividad (Garrard, Lambon Ralph, Hodges \& Patterson, 2001), la dominancia (Ashcraft, 1978), la distancia semántica (Zannino, Perri, Pasqualetti, Di Paola, Caltagirone \& Carlesimo, 2006) y la correlación de atributos (Tyler, Moss, Durrant-Peatield \& Levy, 2000). Estas se han estudiado tanto en sujetos sanos como en población con patologías neurológicas (Gonnerman, Andersen, Devlin, Kempler \& Seidenberg, 1997; Peraita, Díaz \& Anlló-Vento, 2008; Peraita \& Moreno, 2006). Estas variables forman parte de la información que contienen las normas de producción de atributos semánticos y permiten el contraste de hipótesis teóricas. Un ejemplo de este tipo de estudios es el realizado por Gonnerman et al. (1997), quienes aplicaron 
este modelo al deterioro semántico de pacientes con enfermedad de Alzheimer de diferentes grados de evolución y observaron que se dabe a un patrón específico vinculado con la diferencia en la cantidad de atributos intercorrelacionados y distintivos de los dominios de seres vivos y artefactos.

Como respuesta a la problemática de reconocer una diferenciación semántica y lingüística en la producción de atributos de acuerdo a diferentes grupos culturales, en los últimos años se han recolectado normas de producción de atributos semánticos en varios idiomas: inglés (Buchanan, Valentine \& Maxwell, 2019; Devereux, Tyler, Geertzen \& Randall, 2014; McRae et al., 2005; Vinson \& Vigliocco, 2008), holandés (De Deyne et al., 2008; Ruts, De Deyne, Ameel, Vanpaemel, Verbeemen \& Storms, 2004), alemán (Kremer \& Baroni, 2011), español (Vivas, Vivas, Comesaña, García Coni \& Vorano, 2017) y tres versiones en italiano (Kremer \& Baroni, 2011; Lenci, Baroni, Cazzolli \& Marotta, 2013; Montefinese, Ambrosi, Fairfield \& Mammarella, 2013).

Todos estos trabajos fueron realizados sobre muestras de adultos jóvenes universitarios. Sin embargo, diferentes estudios han puesto de manifiesto la existencia de características específicas en el procesamiento semántico de los adultos mayores. En este sentido, hay evidencia, por ejemplo, de que estos usan y procesan el lenguaje de una manera diferente a los individuos más jóvenes (Amerson, Ferraro, Hale \& Lima, 1992). Algunas investigaciones señalan que las dificultades en la recuperación de las palabras son el resultado de la reducción del acceso a las representaciones léxicas y fonológicas (Sommers, 1996). Por otra parte, hay trabajos que indican que el tamaño del lexicón aumenta hasta la edad adulta media y luego disminuye (Schaie \& Willis, 1993). También que la red de asociaciones semánticas, si bien es cualitativamente semejante entre adultos jóvenes y mayores, tiene menor cantidad de asociaciones en estos últimos (Zortea \& Fumagalli de Salles, 2012). Estos hallazgos coinciden con trabajos recientes del Aging Lexicon Consortium (Wulff, De Deyne, Jones, Rui Mata \& the Aging Lexicon Consortium, 2019). A su vez, hay trabajos que reportan una mayor tendencia de los adultos mayores a establecer relaciones temáticas - entre objetos que coocurren en un mismo contexto- - que taxonómicas - entre objetos de la misma categoría semántica-(García, Ison \& Vivas, 2020; Maintenant, Blaye \& Paour, 2011; Pennequin, Fontaine, Bonthoux, Scheuner $\&$ Blaye, 2006) lo que sugiere configuraciones distintas de la organización de los conceptos. También hay estudios realizados a nivel del discurso que sugieren la existencia de algunas diferencias en el tipo de atributo 'sonido' y en la categoría de 'seres vivos' (Kintz \& Wright, 2017). No obstante, parece ser que las representaciones conceptuales que subyacen al significado de un concepto se preservan en la vejez (Thornton \& Light, 2006).

Recientemente, Wulff, de Deyne, Jones, Rui Mata y the Aging Lexicon Consortium (2019) sugirieron que el estudio de los modelos del léxico mental en el envejecimiento debe integrar el estudio interdisciplinario de factores ecológicos, psicológicos y neurales para generar ideas más sólidas y generalizables sobre una base ecológica y computacional del léxico mental que envejece.

Los datos experimentales provenientes de estudios de priming semántico en personas mayores confirman que las conexiones entre representaciones son más ricas y resistentes a los déficits de transmisión en la red semántica (MacKay \& James, 2001). Estudios sobre el caudal léxico de las personas mayores revelan mejor desempeño de estos que de los jóvenes en las pruebas estándares de vocabulario (Kemper \& Sumner, 2001; Lindenberger \& Baltes, 1997). El conocimiento léxico-semántico acumulado durante la vida adulta permanece estable en la edad avanzada, aunque puede observarse un enlentecimiento en el procesamiento semántico de las oraciones relacionado con la construcción de una representación mental del significado (Véliz, Riffo \& Arancibia, 2010). 
Por estas razones resulta de especial interés comprender las características de la producción de atributos semánticos en adultos mayores y establecer la estabilidad de los significados atribuidos a diferentes conceptos en comparación con los adultos jóvenes y, consecuentemente, la continuidad de las representaciones conceptuales que subyacen al significado de un concepto en la vejez.

\section{Fluencia verbal semántica}

Tradicionalmente, la generación verbal se evalúa mediante pruebas de fluencia a partir de solicitar la producción de palabras pertenecientes a un grupo específico dentro de un límite de tiempo. En la tarea fluencia verbal semántica (FVS), la evocación de palabras se fundamenta principalmente en llevar a cabo asociaciones semánticas para luego estudiar la interpretación y clasificación de las mismas. En general, los estudios han encontrado que los adultos mayores tienen un rendimiento menor que los adultos jóvenes en estas tareas (Lanting, Haugrud \& Crossley, 2009). Los estudios en España y Argentina (Perea, Ladera \& Rodríguez, 2005; Butman, Allegri, Harris \& Drake, 2000) parecen sugerir que la edad, los años de escolaridad y el nivel cognitivo son los mejores predictores del rendimiento en estas pruebas.

Existen pocos estudios que comparen sistemáticamente la producción de atributos semánticos en adultos jóvenes y adultos mayores; la falta de antecedentes respecto a estudios que comparen la distancia semántica entre conceptos y la fluencia verbal en poblaciones de jóvenes y mayores de una misma región es lo que conduce al objetivo de analizar y comparar dichos aspectos en la producción de atributos semánticos entre jóvenes y adultos. Pareciera haber evidencia de que existe una estabilidad de los núcleos semánticos compartidos entre lenguas (Kremer \& Baroni, 2011; Vivas, Kogan, Romanelli, Lizarralde \& Corda, 2020; Vivas, Montefinese, Bolognesi \& Vivas, 2020).
Surge de este modo la pregunta: ¿hasta qué punto se sostiene la estabilidad nuclear de significados en un estudio generacional dentro de una misma lengua? La originalidad del presente trabajo consiste en explorar si esta situación se verifica dentro de la misma comunidad lingüística.

Para ello y en el marco de las investigaciones sobre procesamiento de los significados y las representaciones mentales, se aborda la comparación de la producción de atributos semánticos a partir de conceptos de la categoría 'seres vivos' y 'no vivos' de dos grupos etarios: adultos jóvenes y adultos mayores sin patología cognitiva. Particularmente se realiza la comparación entre los conceptos en ambas poblaciones por medio de la técnica de análisis de la distancia semántica entre los atributos para adultos jóvenes y adultos mayores. Complementariamente se realiza el análisis de la fluencia verbal en la producción de atributos semánticos dados por ambos grupos. Las hipótesis básicas suponen que existen diferencias significativas en la producción de atributos en relación a la fluencia verbal semántica y, complementariamente, estabilidad semántica entre los conceptos definidos por ambos grupos etarios.

\section{Metodología}

\section{Diseño y participantes}

Se utilizó un diseño correlacional, descriptivo, cuasi experimental. La muestra de adultos mayores fue no probabilística y estuvo conformada por 90 participantes de Mar del Plata y su zona de influencia, mayores de 65 y menores de 90 años, sin patologías psiquiátrica o neurológica, ni DCL. Media $=72.97$, DS $=6.98$, rango entre 60 y 89 , moda $=70$, el $73.23 \%$ fueron mujeres. Los datos de adultos jóvenes fueron tomados de las Normas de Producción de Atributos Semánticos en Español para 400 conceptos concretos (Vivas et al., 2017), compuesto básicamente por 
estudiantes universitarios de entre 20 y 40 años de edad. Media $=24.01, \mathrm{DS}=4.05$, rango entre $19 \mathrm{y}$ 38, moda $=23$, el $73 \%$ fueron mujeres. Los 130 conceptos extractados de las Normas de Adultos Jóvenes se corresponden con 120 participantes cada concepto en las normas fue respondido por 30 participantes - Los grupos se homologaron respecto a género y nivel socioeducativo. Los participantes del grupo de adultos mayores fueron seleccionados intencionalmente, todos ellos concurrentes al Programa de Adultos Mayores de la Universidad Nacional de Mar del Plata. Su composición habitual es la de personas que se jubilaron de la Universidad o sectores vinculados a la misma. Se consideraron los siguientes criterios de inclusión: cada participante fue hablante español argentino nativo, con educación primaria completa o superior y con un nivel socioeconómico medio-alto. El nivel socioeducativo se calculó utilizando el Índice de Hollingshead (2011) para lo cual se categorizó, en primer lugar, el nivel educativo según la escala de siete puntos (Pascual, Galperín \& Bornstein, 1993) en función del sistema educativo argentino. En segundo lugar, se categorizó el nivel ocupacional según la Escala de Grupos Ocupacionales (EGO 70) de Sautú (1989) elaborada para población argentina, basada en el Código de Ocupaciones del INDEC - Instituto Nacional de Estadística y Censos de la República Argentina- del año 2018, que responde a la Clasificación Internacional Uniforme de Ocupaciones (CIUO) de la Oranización Internacional del Trabajo (OIT) de 1986. Otro criterio de inclusión fue tener visión normal o corregida a normal. Previo a la realización de las tareas se solicitó el consentimiento informado escrito. Este estudio se rige por el reglamento del Comité de Ética del Consejo Nacional de Investigaciones Científicas y Técnicas (Conicet), Res. 613/05, el artículo 18 de la Ordenanza de Consejo Superior (OCS 136/08) de la Universidad Nacional de Mar del Plata (UNMDP), por la Resolución Ministerial 1480/11 y la Ley Provincial 11044/09.

\section{Materiales}

Se extrajeron 130 conceptos con sus correspondientes atributos de los 400 presentados en las Normas de Producción de Atributos Semánticos en Español para 400 conceptos concretos (Vivas et al., 2017). Esta selección se realizó en base a su representatividad categorial en el total de las normas. Contienen 12 categorías dentro de los dominios 'vivos' (65) y 'no-vivos' (65), discriminados del siguiente modo:

Dominio vivos: Animales (48) y Frutas y verduras (17).

Dominio no-vivos: Instrumentos musicales (9), Indumentaria (9), Vehículos (11), Accesorios (7), Utensilios (9), Muebles (6), Herramientas (6), Construcciones (4), Objetos (1) y Contenedores (3).

\section{Procedimiento}

En adultos mayores se utilizó la tarea FLT (Feature Listing Task), que básicamente consiste en solicitar la enumeración libre de propiedades que caracterizan un concepto presentado. La administración fue individualizada, mediante condiciones estandarizadas y sistemáticas, en una sesión de 20 minutos. Primeramente, se detalló el objetivo e importancia del estudio y se presentaron ejemplos (véase anexo 1).

Para los fines del procesamiento informático de los mismos fue necesario realizar un proceso de unificación respecto a la variabilidad sintáctica sin perjuicio de la variabilidad semántica, de los atributos dados por ambas poblaciones. Las normas de producción de rasgos semánticos proporcionan muchas medidas cuantitativas de diferentes características y variables del concepto que son necesarias para resolver algunos debates en torno a la naturaleza de la organización tanto normal, como patológica de la memoria semántica. Estas normas consisten en colecciones empíricas de características que las personas utilizan para describir los conceptos. La unifica- 
ción de atributos se realizó según los criterios de registro propuestos por McRae et al. (2005). Se generó un vector $n$-dimensional con los atributos descriptores para cada uno de los 130 conceptos en ambas poblaciones. Cada vector se construyó con el software Definition Finder (Vivas et al., 2014). Los resultados se expresan en valores numéricos que fluctúan entre 0 y 1 , donde 1 es el mayor peso. Para la obtención de las distancias se desarrolló el software Synonym Finder, disponible en http://iaai.fi.mdp.edu.ar:8080/sfweb. Se usó la técnica geométrica de comparación de vectores en el espacio euclidiano $n$-dimensional usual, a partir del ángulo formado entre los mismos. Este paralelismo representa el caso de mayor semejanza; la ortogonalidad, el de mayor diferencia (Kintsch, 2001). La medida utilizada para calcular la relación semántica es el coseno entre vectores. Esta medida puede ser considerada análoga a los coeficientes de correlación, cuanto más relacionadas semánticamente están dos palabras, mayor es el valor de su coseno. De este modo, se calculó la distancia semántica entre los 260 conceptos bajo estudio (130 para cada población). Sobre esta matriz se realizó un análisis de clúster jerárquico con el método de Johnson (1967) por medio del software NetDraw (Borgatti, 2002). De este modo se generaron los agrupamientos por mayor cohesión (menor distancia) entre subgrupos y su relación con la totalidad. El gráfico muestra el agrupamiento jerárquico de los conceptos para ambos grupos etarios tanto en relación a los atributos correspondientes a conceptos dados por ambos grupos, como a la de los atributos correspondientes tanto a conceptos de seres vivos como no vivos, de manera que la existencia de proximidad semántica se interpretara como representaciones mentales similares de los conceptos.

Respecto al análisis de la fluencia semántica, según el modo de recolección empírica de los atributos, se optó por realizar la sumatoria de toda la producción de atributos dados como definidores de conceptos pertenecientes a las categorías de seres vivos y no vivos, diferenciando la producción de cada grupo etario para poder compararlos luego. El antecedente para analizar la FVs de este modo se remite a un estudio que compara la riqueza semántica entre hablantes monolingües y hablantes bilingües (Taler, Zunini \& Kousaie, 2016).

\section{Resultados}

Primeramente, para la obtención de los vectores se procedió a unificar las respuestas dadas para los 130 conceptos por el grupo de Adultos Mayores (AM). A modo de ejemplo, se muestra la obtención de los atributos para el concepto: 'hiena'. La unificación se realizó siguiendo las Normas de Producción de Atributos Semánticas en Español (Vivas et al., 2017), confeccionadas para adultos jóvenes (AJ). A modo de ejemplo el concepto 'hiena' se unificó de la manera como se muestra en la tabla 1.

Nótese que atributos como tiene_cuatro_patas y es_cuadrupedo corresponden a diferentes categorías de respuesta. Desde MacRae et al. (2005) se han refinado las metodologías de unificación y categorización. En este trabajo usamos las de Wu y Barsalou (2009); así, por ejemplo, tiene_cuatro_patas se clasifica como partonómico cuantificado, mientras que es_cuadrupedo es un categorial superordenado. Ambos corresponden a categorías distintas e involucran distintos circuitos cerebrales. Una vez unificados los datos, estos fueron cargados y procesados en el Definition Finder. La tabla 2 muestra un extracto de los resultados obtenidos en la construcción de los vectores para el concepto 'hiena' del dominio 'seres vivos'.

De esta manera, se observa cómo se genera un peso relativo para cada atributo de acuerdo a la frecuencia del mismo y su ponderación según la posición que ocupa el atributo en la producción individual de cada grupo. El total de atributos para ese concepto forma un vector $n$-dimensional. Así 
Tabla 1.

Unificación del concepto "hiena"

\begin{tabular}{|c|c|c|c|c|}
\hline $\begin{array}{c}\text { Participante } \\
\text { Sexo, Edad, } \\
\text { Facultad }\end{array}$ & Atributo 1 & Atributo 2 & Atributo 3 & Atributo 4 \\
\hline $1,23,1$ & SE_RÍE & ES_LIGERO & MUERDE & ES_CARROÑERA \\
\hline $1,22,1$ & ES_UN_ANIMAL & SE_RÍE & $\begin{array}{l}\text { PUEDE_SER_-} \\
\text { PERRO_SALVAJE }\end{array}$ & VIVE_EN_LA_SELVA \\
\hline $2,24,3$ & ES_FEO & ES_SALVAJE & VIVE_EN_MANADA & \\
\hline $1,25,1$ & ES_UN_ANIMAL & ES_SALVAJE & ES_CARNÍVORO & VIVE_EN_MANADA \\
\hline $1,26,4$ & ES_FEROZ & SE_RÍE & TIENE_PELO & ES_MARRÓN \\
\hline $1,24,1$ & ES_MALO & & & \\
\hline $1,32,1$ & ES_UN_ANIMAL & TIGRE & ES_FEROZ & \\
\hline $2,23,4$ & ES_UN_ANIMAL & & & \\
\hline $1,27,1$ & ES_UN_ANIMAL & VÍBORA & & \\
\hline $1,32,1$ & ES_UN_ANIMAL & & & \\
\hline $1,27,2$ & ES_UN_ANIMAL & ES_SALVAJE & ES_TRAICIONERO & ES_FEO \\
\hline $1,26,3$ & VIVE_EN_LA_SELVA & ES_AGRESIVO & SE_RÍE & ES_MORTAL \\
\hline $2,34,4$ & ES_MALO & ES_CARNICERO & MATA & SE_RIE \\
\hline $2,32,4$ & ES_UN_ANIMAL & ES_SALVAJE & $\begin{array}{l}\text { TIENE_OLOR_- } \\
\text { DESAGRADABLE }\end{array}$ & ATACA \\
\hline $1,25,1$ & ES_UN_ANIMAL & ES_DESPRECIABLE & & \\
\hline $1,24,1$ & SE_RÍE & ES_FEROZ & ES_SALVAJE & ATACA \\
\hline $1,20,1$ & SE_RÍE & ES_UN_ANIMAL & & \\
\hline $1,19,1$ & ES_CARNICERO & ES_CANINO & ES_OVERO & ES_SALVAJE \\
\hline $1,23,1$ & ES_REPULSIVO & SE_RÍE & NO_LA_QUIERO & \\
\hline $1,27,2$ & VIVE_EN_ÁFRICA & ES_SALVAJE & & \\
\hline $2,24,3$ & ES_UN_ANIMAL & & & \\
\hline $2,23,3$ & ES_FEO & SE_RÍE & ES_UN_ANIMAL & ES_PELUDO \\
\hline $1,23,2$ & ES_UN_ANIMAL & SE_RÍE & ES_FEO & VIVE_EN_ÁFRICA \\
\hline $1,22,1$ & ES_UN_ANIMAL & ES_PELUDO & SE_RÍE & \\
\hline $1,23,1$ & VIVE_EN_ÁFRICA & ES_UN_ANIMAL & ES_PELUDO & ES_FEO \\
\hline $1,29,1$ & ES_UN_ANIMAL & ES_PELUDO & TIENE_PATAS & TIENE_CUATRO_PATAS \\
\hline $1,24,2$ & ES_FEO & ES_UN_ANIMAL & NO ME GUSTA & \\
\hline $1,23,1$ & ES_UN_ANIMAL & TIENE_OLOR_FEO & & \\
\hline $2,26,4$ & ES_UNA_FIERA & ES_MALO & & \\
\hline $1,23,1$ & ES_UN_ANIMAL & $\begin{array}{l}\text { ES_PERSONA_- } \\
\text { DESAGRADABLE }\end{array}$ & & \\
\hline
\end{tabular}


se obtuvieron 130 vectores para cada uno de los grupos etarios. Luego se procedió a producir una comparación inter e intraedad para los 130 vectores de cada población. Se obtuvo una matriz cuadrada de $260 \times 260$ conceptos. En la figura 1 se presenta un extracto de la misma.

Tabla 2.

Resultados de los valores arrojados por el software Definition Finder para la palabra 'hiena'en adultos mayores y en adultos jóvenes. Los valores fluctúan entre 0 y 1

\begin{tabular}{|c|c|c|c|}
\hline Adultos mayores & & Adultos jóvenes & \\
\hline Atributo & Valor & Atributo & Valor \\
\hline ES_UN_ANIMAL & .55 & ES_UN_ANIMAL & .71 \\
\hline ES_SALVAJE & .15 & SE_RÍE & .33 \\
\hline ES_MALO & .08 & ES_UN_MAMÍFERO & .13 \\
\hline ES_FEROZ & .07 & ES_MALO & .09 \\
\hline ES_CARNICERO & .06 & ES_UN_CARNÍVORO & .09 \\
\hline $\begin{array}{l}\text { TIENE_OLOR_- } \\
\text { DESAGRADABLE }\end{array}$ & .03 & ES_GRIS & .06 \\
\hline ATACA & .02 & RUGE & .06 \\
\hline ES_CHICO & .01 & TIENE_PELO & .06 \\
\hline SE_RÍE & .28 & COME_CARROÑA & .06 \\
\hline ES_FEO & .13 & REY_LEÓN & .05 \\
\hline ES_PELUDO & .08 & ES_FEROZ & .05 \\
\hline VIVE_EN_ÁFRICA & .07 & TIENE_CUATRO_PATAS & .04 \\
\hline VIVE_EN_LA_SELVA & .05 & ES_SALVAJE & .04 \\
\hline VIVE_EN_MANADA & .02 & ES_AGRESIVO & .04 \\
\hline \multirow[t]{9}{*}{ ES_CARROÑERO } & .01 & TIENE_DIENTES & .04 \\
\hline & & VIVE_EN_MANADA & .04 \\
\hline & & ES_PELIGROSO & .04 \\
\hline & & ES_CUADRÚPEDO & .03 \\
\hline & & PARECE_A_UN_PERRO & .03 \\
\hline & & TIENE_MANCHAS & .03 \\
\hline & & TIENE_PATAS & .02 \\
\hline & & VIVE_EN_NATURALEZA & .02 \\
\hline & & VIVE_EN ÁFRICA & .02 \\
\hline
\end{tabular}


Figura 1.

Extracto de la matriz cuadrada de distancias entre los conceptos según grupos etarios

\begin{tabular}{|c|c|c|c|c|c|c|c|c|c|c|c|c|c|}
\hline & 4 & 1 & c & s & E & s & 6 & $H$ & 1 & $y$ & $\varepsilon$ & i & N \\
\hline 1 & 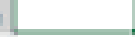 & ABASCOAN & Aasvocosa & ACOEDEONAS & A ACOSOECON A & ACULAA & ACULA AM & ANANAN & ANCWAAN & ANCUILA & ANOUEUAAN & AnON & APOAN \\
\hline 2 & LANDCON & 10 & 0.615 & o.003 & 0.008 & 0.000 & 0,000 & 0.003 & 2001 & 0.000 & 2000 & 0,000 & 0.000 \\
\hline 3 & Nawnco MM & 10655 & 1.0 & o.cos & 0.012 & 0.000 & 0000 & 0.000 & 0.000 & 0,000 & 2000 & 0.000 & 9000 \\
\hline 4 & ACORDECN A & Ne. Dos & 0,005 & 10 & 0.700 & 0.002 & 0.006 & 0.000 & 0.001 & 0,000 & 2000 & 0,000 & 9.000 \\
\hline 5 & ACOSCOCON-A & wedos & 0.012 & 6.25 & 10 & 0.000 & 0,000 & 0.000 & 0,000 & 0,000 & 2000 & 0,000 & 9.000 \\
\hline 5 & 5 AGUEANA & 0.000 & 0,000 & $\cos 2$ & 0,000 & 1.0 & a.s74 & 0.000 & 9005 & 0.15n & acse & 0,000 & 9000 \\
\hline 7 & AGULA.AMI & 0.000 & 0000 & 0.006 & 0,000 & ons & 20 & 0.000 & a.anz & 0.155 & 0.081 & oxca & 0.000 \\
\hline 1 & ANANAN & exas & 0,000 & 0.000 & 0,000 & 0.000 & 0,000 & 1.0 & a.35T & 0,000 & ats & 0004 & estu \\
\hline 9 & - Axaserase & s.sot & 0000 & obet & 0000 & boes & adat2 & 0037 & to & 0000 & ats & acos & eass \\
\hline ne & - AVCULAa & 5000 & 0.000 & 0000 & 0000 & $0.13 t$ & a.ts & 0.000 & $a 000$ & 1.0 & a.xo4 & 0002 & $\sec 2$ \\
\hline$\pi$ & 1 ANCULA AN & 6000 & 0.000 & 6000 & 0000 & 60.058 & acosi & 0.007 & aats & 0.04 & 20 & 0.036 & est \\
\hline ga & 2 ANON & 0000 & 0,000 & 0.000 & 0,000 & 0.000 & 0.004 & 0.004 & a.at 7 & 0.027 & 0.236 & 1.0 & esos \\
\hline ] & 1 ANO as & 0000 & 0,000 & 0,000 & 0,000 & 0.000 & 0,000 & 0.064 & a.ass & 0.002 & Qset & axes & 18 \\
\hline 4 & 4 Nakin & 0.006 & 0.000 & 0.002 & 0.000 & 0.001 & 0.200 & 0.000 & 0.004 & 0.153 & 0.501 & oous & 9000 \\
\hline 15 & 5 saklam & 0.009 & 0,000 & 0.002 & 0.000 & 0.095 & 0.071 & 0.000 & 0005 & 0.085 & 2061 & 0.000 & 2000 \\
\hline 14 & 4 Naseuans & $\cos s$ & 9000 & 0.000 & 0,000 & $0.25 t$ & 0.200 & 0.000 & 0,000 & 0590 & $0: 35$ & 0,000 & 0.000 \\
\hline 19 & 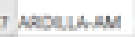 & enzt & 0,000 & 0,000 & 0,000 & 0.005 & 0061 & 0.000 & 0,000 & 0.179 & 0056 & 0,000 & 0.000 \\
\hline
\end{tabular}

Se puede observar en este corte de la matriz simétrica, que se han resaltado en negrita, los valores de proximidad para el mismo concepto dado para jóvenes y para mayores. Por ejemplo, para el mismo concepto 'anguila' entre mayores y jóvenes se observa una distancia de .704. Asimismo, se puede observar como también se generan distancias por debajo de .5 como en el caso de 'águila' en mayores y 'ardilla' en jóvenes con .2, que marca un valor para conceptos en donde los atributos han diferido. También se observa para esos mismos conceptos, pero esta vez dentro de la misma población de jóvenes, una distancia de 2. Se muestran en la tabla 3 los valores de la matriz coseno para los mismos conceptos en función de los grupos de edad.

El promedio de las distancias es de .73. Su distribución se puede apreciar en los siguientes valores: de 0 a .5 el $10 \%$, de .5 a .7 el $28 \%$ y más del .7 el $62 \%$.

Tabla 3.

Coseno para los mismos conceptos en función de los grupos de edad

\begin{tabular}{lccccc}
\hline Concepto & Coseno & Concepto & Coseno & Concepto & Coseno \\
\hline Abanico & .615 & Cisne & .769 & Mosca & .831 \\
\hline Acordeón & .702 & Cocodrilo & .834 & Moto & .671 \\
\hline Águila & .874 & Colador & .46 & Murciélago & .778 \\
\hline Ananá & .937 & Colectivo & .732 & Olla & .255 \\
\hline Anguila & .704 & Collar & .636 & Oso & .884 \\
\hline Apio & .905 & Conejo & .777 & Pala & .652 \\
\hline
\end{tabular}




\begin{tabular}{|c|c|c|c|c|c|}
\hline Concepto & Coseno & Concepto & Coseno & Concepto & Coseno \\
\hline Araña & .862 & Corbata & .935 & Palta & .814 \\
\hline Ardilla & .552 & Corona & .722 & Pantalón & .881 \\
\hline Armario & .914 & Cucaracha & .848 & Paraguas & .929 \\
\hline Armónica & .721 & Cuchara & .76 & Pato & .559 \\
\hline Arpa & .846 & Cucharón & .699 & Perro & .469 \\
\hline Auto & .686 & Cuchillo & .828 & Piano & .865 \\
\hline Avestruz & .48 & Delfín & .849 & Pimiento & .916 \\
\hline Avión & .413 & Destornillador & .564 & Pingüino & .497 \\
\hline Ballena & .776 & Durazno & .749 & Pirámide & .869 \\
\hline Banana & .392 & Elefante & .833 & Pollera & .827 \\
\hline Bicicleta & .637 & Escarabajo & .645 & Pulpo & .668 \\
\hline Blusa & .664 & Espátula & .575 & Rastrillo & .537 \\
\hline Bol & .461 & Flauta & .489 & Ratón & .731 \\
\hline Bota & .652 & Frasco & .71 & Sapo & .865 \\
\hline Botella & .753 & Frutilla & .944 & Sartén & .648 \\
\hline Búfalo & .88 & Gallina & .748 & Saxofón & .91 \\
\hline Burro & .817 & Gallo & .787 & Silla & .611 \\
\hline Caballo & .775 & Gato & .628 & Sillón & .843 \\
\hline Cabra & .806 & Granero & .501 & Tenedor & .779 \\
\hline Cadena & .586 & Guante & .561 & Tigre & .802 \\
\hline Cama & .457 & Guitarra & .786 & Tijera & .935 \\
\hline Camello & .869 & Hacha & .805 & Tomate & .525 \\
\hline Camión & .727 & Helicóptero & .386 & Tornillo & .52 \\
\hline Camisa & .866 & Hiena & .899 & Tortuga & .924 \\
\hline Campera & .543 & Hormiga & .51 & Tractor & .816 \\
\hline Cangrejo & .634 & Iglesia & .418 & Tren & .89 \\
\hline Caracol & .853 & Jirafa & .907 & Trineo & .882 \\
\hline Casa & .546 & Lechuga & .683 & Trombón & .953 \\
\hline Cebolla & .728 & Lechuza & .723 & Trompeta & .724 \\
\hline Cebra & .857 & León & .658 & Uvas & .608 \\
\hline Cereza & .916 & Limón & .93 & Vaca & .59 \\
\hline Chaleco & .853 & Loro & .896 & Velero & .751 \\
\hline Chancho & .689 & Manzana & .9 & Vestido & .809 \\
\hline Choclo & .841 & Mariposa & .764 & Víbora & .651 \\
\hline Ciervo & .836 & Martillo & .68 & Zanahoria & .719 \\
\hline Cigüeña & .774 & Mecedora & .447 & Zapato & .581 \\
\hline \multirow[t]{2}{*}{ Cinturón } & .699 & Mesa & .716 & Zorrino & .95 \\
\hline & & & & Zorro & .887 \\
\hline
\end{tabular}


Luego, con la matriz coseno completa se procedió a hacer un análisis de clúster utilizando la técnica de Johnson (1967) - figura 2-. En este caso, para facilitar la visualización se exigió que los clústers se agrupasen con una distancia menor a .5.

En la figura se puede observar cómo se cohesionan los conceptos con este nivel de proximidad entre mayores y jóvenes. Se observa cómo se agrupan y delimitan las diferentes categorías, por ejemplo, frutas, medios de transporte, indumentaria, instrumentos musicales, herramientas, etc.; se distingue con flechas la distancia semántica entre los conceptos. La proximidad semántica y la agrupación de los conceptos se interpretan como representaciones mentales similares. Se observa mayor cohesión para los conceptos arpa, acordeón, saxofón, piano, trompeta. Dicha agrupación es atribuible a las características de dichos conceptos ya que son seres no vivos e instrumentos musicales. También se agrupan lechuga, apio, cebolla, y, por otro lado, búfalo, elefante, oso en la categoría de 'verduras' los primeros y 'animales' los segundos. Las categorías a su vez, se agrupan de acuerdo a los dominios 'seres vivos' y 'no vivos'.

Figura 2.

Ploteo luego del escalamiento multidimensional de las agrupaciones emergentes del análisis de cluster entre conceptos correspondientes a ambos grupos etarios: adultos jóvenes (JV) y adultos mayores (MY)

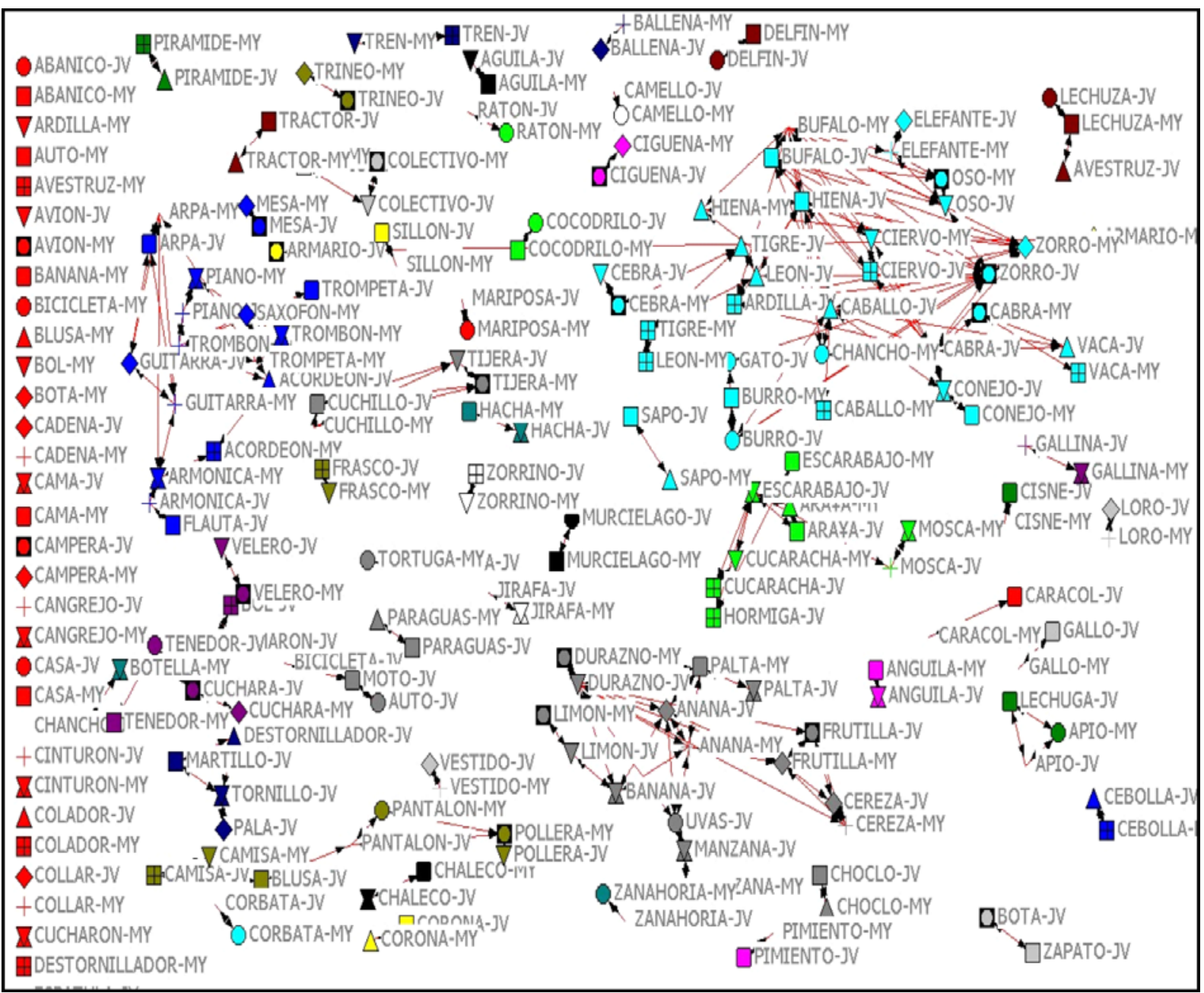


En cuanto al estudio de fluencia, se procedió a realizar el recuento del total de los atributos producidos por cada uno de los 130 conceptos para la población de AM y se los comparó con los atributos que produjo para esos mismos conceptos la población de AJ. Se muestra en la tabla 4 la cantidad de atributos que produjeron ambos grupos etarios para los 130 conceptos.

Tabla 4.

Producción de atributos para cada concepto en ambos grupos etarios

\begin{tabular}{|c|c|c|c|c|c|c|c|c|}
\hline Concepto & $\mathrm{AM}$ & $\mathrm{AJ}$ & Concepto & AM & $\mathrm{AJ}$ & Concepto & $\mathrm{AM}$ & AJ \\
\hline Abanico & 136 & 116 & Cisne & 156 & 106 & Mosca & 113 & 182 \\
\hline Acordeón & 104 & 173 & Cocodrilo & 165 & 126 & Moto & 74 & 171 \\
\hline Águila & 127 & 148 & Colador & 102 & 145 & Murciélago & 90 & 175 \\
\hline Ananá & 172 & 223 & Colectivo & 169 & 179 & Olla & 29 & 97 \\
\hline Anguila & 91 & 230 & Collar & 135 & 98 & Oso & 169 & 102 \\
\hline Apio & 122 & 149 & Conejo & 124 & 123 & Pala & 107 & 80 \\
\hline Araña & 159 & 143 & Corbata & 135 & 129 & Palta & 160 & 79 \\
\hline Ardilla & 130 & 191 & Corona & 150 & 155 & Pantalón & 125 & 69 \\
\hline Armario & 138 & 191 & Cucaracha & 158 & 125 & Paraguas & 141 & 158 \\
\hline Armónica & 106 & 187 & Cuchara & 134 & 166 & Pato & 103 & 125 \\
\hline Arpa & 127 & 177 & Cucharon & 108 & 167 & Perro & 142 & 149 \\
\hline Auto & 158 & 186 & Cuchillo & 146 & 195 & Piano & 151 & 110 \\
\hline Avestruz & 163 & 119 & Delfín & 178 & 130 & Pimiento & 89 & 116 \\
\hline Avión & 102 & 155 & Destornillador & 124 & 76 & Pingüino & 108 & 144 \\
\hline Ballena & 151 & 122 & Durazno & 100 & 108 & Pirámide & 95 & 179 \\
\hline Banana & 157 & 190 & Elefante & 80 & 73 & Pollera & 179 & 164 \\
\hline Bicicleta & 167 & 136 & Escarabajo & 72 & 96 & Pulpo & 152 & 187 \\
\hline Blusa & 77 & 156 & Espátula & 105 & 165 & Rastrillo & 123 & 52 \\
\hline Bol & 150 & 179 & Flauta & 137 & 134 & Ratón & 125 & 79 \\
\hline Bota & 125 & 210 & Frasco & 102 & 152 & Sapo & 159 & 71 \\
\hline Botella & 90 & 149 & Frutilla & 155 & 136 & Sartén & 140 & 137 \\
\hline Búfalo & 119 & 140 & Gallina & 165 & 126 & Saxofón & 140 & 151 \\
\hline Burro & 141 & 112 & Gallo & 132 & 146 & Silla & 156 & 127 \\
\hline Caballo & 200 & 177 & Gato & 107 & 125 & Sillón & 154 & 143 \\
\hline Cabra & 146 & 148 & Granero & 103 & 148 & Tenedor & 112 & 129 \\
\hline Cadena & 151 & 153 & Guante & 105 & 102 & Tigre & 129 & 142 \\
\hline Cama & 95 & 146 & Guitarra & 134 & 179 & Tijera & 118 & 140 \\
\hline
\end{tabular}




\begin{tabular}{|c|c|c|c|c|c|c|c|c|}
\hline Concepto & $\mathrm{AM}$ & $\mathrm{AJ}$ & Concepto & AM & $\mathrm{AJ}$ & Concepto & AM & $\mathrm{AJ}$ \\
\hline Camello & 94 & 135 & Hacha & 86 & 117 & Tomate & 116 & 19 \\
\hline Camión & 115 & 188 & Helicóptero & 125 & 142 & Tornillo & 140 & 136 \\
\hline Camisa & 153 & 133 & Hiena & 100 & 129 & Tortuga & 172 & 169 \\
\hline Campera & 107 & 155 & Hormiga & 105 & 120 & Tractor & 140 & 117 \\
\hline Cangrejo & 93 & 151 & Iglesia & 71 & 162 & Tren & 172 & 109 \\
\hline Caracol & 130 & 141 & Jirafa & 120 & 121 & Trineo & 82 & 166 \\
\hline Casa & 189 & 167 & Lechuga & 139 & 135 & Trombón & 142 & 147 \\
\hline Cebolla & 152 & 159 & Lechuza & 112 & 115 & Trompeta & 125 & 210 \\
\hline Cebra & 127 & 172 & León & 140 & 147 & Uvas & 140 & 117 \\
\hline Cereza & 145 & 165 & Limón & 159 & 145 & Vaca & 172 & 132 \\
\hline Chaleco & 98 & 161 & Loro & 90 & 132 & Velero & 102 & 151 \\
\hline Chancho & 135 & 175 & Manzana & 112 & 172 & Vestido & 146 & 116 \\
\hline Choclo & 155 & 178 & Mariposa & 158 & 142 & Víbora & 99 & 133 \\
\hline Ciervo & 73 & 189 & Martillo & 160 & 137 & Zanahoria & 146 & 137 \\
\hline Cigüeña & 152 & 138 & Mecedor & 139 & 125 & Zapato & 108 & 222 \\
\hline \multirow[t]{2}{*}{ Cinturón } & 129 & 197 & Mesa & 171 & 116 & Zorrino & 105 & 145 \\
\hline & & & & & & Zorro & 136 & 100 \\
\hline
\end{tabular}

Con los datos así obtenidos, se realizó el Test de Shapiro-Wilk y se observó que la distribución de los valores de la variable dependiente es normal en ambos casos, el nivel de medición de la variable es de razón y el Test de Homogeneidad de varianzas de Levene dio varianza homogénea y dispersión similar en sus distribuciones; por ello se realizó un test de medias para muestras independientes.

El valor medio de la cantidad de atributos producidos por los AM resultó ser de 128.80 (DT= 29.30) y de $142.78(\mathrm{DT}=35.11)$ para AJ, lo cual representa un $10 \%$, aproximadamente, a favor de los jóvenes. A partir de estos datos y utilizando una prueba para muestras independientes se realizaron los cálculos de confiabilidad. Así se estimó el error estándar (4.011) y el intervalo [-6.07; -21.86] para la diferencia de medias con un nivel de confian- za de .99. La diferencia de medias entre ambos grupos resultó estadísticamente significativa [ $t=$ 3.48, (258), $p<.01, g l=129]$.

\section{Discusión}

Este estudio se orientó a analizar comparativamente el nivel de estabilidad en la producción de atributos semánticos y, complementariamente, la diferencia en la fluencia verbal semántica entre dos grupos etarios: AJ y AM. El análisis de los datos obtenidos revela que, respecto a la distancia semántica de los 130 conceptos comparados entre ambas poblaciones etarias, la distancia es baja y por ende la similitud es alta. Una distancia promedio mayor a .7 confirma la hipótesis respecto a la estabilidad esperada en la producción de atributos 
nucleares entre los conceptos dados por ambos grupos etarios.

La hipótesis básica de este estudio supone la existencia de una estabilidad semántica entre la producción de atributos para conceptos de las categorías 'seres vivos' y 'no vivos', de AJ y AM de la misma comunidad lingüística. Los resultados de la comparación y el análisis de la distancia semántica entre la producción de atributos semánticos enlistados en cada uno de los 260 vectores, correspondiente al grupo de AJ y al grupo de AM, y el análisis de clúster indican que existe una significativa proximidad semántica entre ellos (más del $80 \%$ con $\mathrm{r}>$.6). Por la naturaleza de la técnica de comparación dicha proximidad semántica se interpreta como indicador de representaciones mentales similares de los conceptos y sugiere un alto grado de similitud en el núcleo del significado para ambos grupos etarios.

La similitud o proximidad semántica que se visualiza entre los vectores de AJ y AM expresa la poca variación en la organización de la memoria semántica, es decir, las representaciones mentales de los conceptos definidos por los mayores continúan cohesionados en las categorías correspondientes, lo que evidencia la estabilidad de los núcleos semánticos en ambos grupos etarios. Según Vivas et al. (2018), los atributos que describen un concepto podrían agruparse en tres niveles: 1) un nivel integrado por muy pocos atributos que se considerarían necesarios para definirlo, los cuales constituirían el núcleo semántico del concepto y son compartidos por una comunidad lingüística; 2) otro conjunto de atributos semánticos que caracterizan al concepto, que se consideran parcialmente compartidos y pueden o no ser producidos por integrantes de dicha comunidad, aunque son reconocidos como válidos por todos, y 3) otros atributos que no son compartidos pues son productos idiosincráticos y particulares de cada individuo.

Ahora bien, los resultados obtenidos con relación al análisis de fluencia muestran una producción de atributos en la población de AM en una magnitud significativamente menor que en la de AJ confirman los reportes que describen algunos estudios previos (Lanting, Haugrud \& Crossley, 2009).

Considerando el hallazgo de la estabilidad semántica entre los dos grupos etarios de la muestra estudiada y en concordancia con la evidencia empírica en la literatura científica, que demuestra que el envejecimiento cognitivo no implica inexorablemente el deterioro en todas las habilidades cognoscitivas de la misma manera ni con la misma dirección (Fernández Ballesteros, Zamarrón, Calero \& Tárraga, 2007; Lövden, Bäckman, Lindenberger, Scahefer \& Schiedeck, 2010), los datos de este estudio revelan la estabilidad semántica entre AJ y AM, y que estos conservan y mantienen la capacidad de definir conceptos y la constitución semántica entre los conceptos, la cual no varía significativamente de la de los AJ.

En este sentido, los resultados de este estudio se corresponden con la evidencia de un funcionamiento general regenerativo conservado y cierta plasticidad neuronal y cognitiva en personas mayores sanas (Navarro \& Calero, 2009). Cabe señalar que los investigadores en neurociencias cognitivas defienden la tesis de la multidimensionalidad y plasticidad del sistema cognitivo durante todo el curso vital, considerando el interjuego entre los factores neurobiológicos y contextuales (Álvarez González \& Trápaga Ortega, 2013; Redolar, 2014). Existe evidencia de que ciertas habilidades de la inteligencia cristalizada, la memoria procedimental y semántica, la reserva cognitiva, la experticia y la sabiduría tienden a conservarse e incluso optimizarse con el avance de la edad (Ardelt \& Edwards, 2015; Arenaza-Urquijo \& Bartréz Faz, 2014; Krzemien \& Richard's, 2011). Las investigaciones actuales desde las neurociencias cognitivas han descubierto los efectos positivos de la plasticidad neuronal en la actividad del cerebro adulto y envejecido (Redolar, 2014), que produce cambios morfológicos, estructurales y funcionales aun en la vejez, que diversifican, coordinan y compensan el sistema neurocognitivo de manera que el AM puede 
continuar respondiendo de manera adaptativa a diversas tareas de demanda cognitiva.

En definitiva, los resultados de este estudio contribuyen a la investigación gerontológica contemporánea centrada en el potencial neurocognitivo, que subordina la perspectiva tradicional centrada en el estudio de la patología neurodegenerativa. Los avances paradigmáticos y metodológicos en la evaluación de las capacidades cognitivas de AM han cuestionado el deterioro cognitivo como un rasgo universal e irreversible, que supera el énfasis en el criterio que vinculaba la competencia cognitiva principalmente a la edad cronológica y a factores biogénicos para incluir la influencia de factores culturales en la competencia cognitiva.

Dado el creciente interés en el estudio de la producción semántica y las variaciones en la fluencia verbal en grupos de una misma comunidad lingüística, este estudio aporta a la cuestión escasamente explorada de la producción de atributos semánticos entre AJ y AM de una misma región y cultura.

\section{Referencias}

Álvarez González, M. A., \& Trápaga Ortega, M. (2013). Principios de neurociencia para psicólogos. Buenos Aires: Paidós.

Amerson, J., Ferraro, F. R., Hale, S., \& Lima, S. D. (1992). General slowing in semantic priming and word recognition. Psychology and Aging, 7, 257-270.

Ardelt, M., \& Edwards, C.A. (2015). Wisdom at the end of life: An analysis of mediating and moderating relations between wisdom and subjective well-being. The Journals of Gerontology. Series B, Psychological Sciences and Social Sciences, 71(3), 502-13. https://doi.org/10.1093/geronb/ gbv051

Arenaza-Urquijo, M., \& Bartréz Faz, D. (2014). Reserva Cognitiva. En D. Redolar(Ed.), Neurociencia cognitiva (pp. 185-197). Madrid: Editorial Médica Panamericana.
Ashby, F. G., \& Alfonso-Reese, L. A. (1995). Categorization as probability density estimation. Journal of Mathematical Psychology, 39(2), 216-233.

Ashcraft, M. H. (1978). Feature dominance and typicality effects in feature statement verification. Journal of Verbal Learning and Verbal Behavior, 17, 155-164.

Baroni, M., Barbu, E., Murphy, B., \& Poesio, M. (2010). Strudel: A distributional semantic model based on properties and types. Cognitive Science, 34(2), 222-254.

Bolognesi, M., \& Steen, G. (2019). Perspectives on abstract concepts: Cognition, language and communication. Netherlands: John Benjamins Publishing Company.

Borgatti, S.P. (2002). NetDraw: Graph visualization soft. Harvard: Analytic Tech.

Buchanan, E., Valentine, K., \& Maxwell, N. (2019). English semantic feature production norms: An extended database of 4436 concepts. Behavior Research Methods. https://doi. org/10.3758/s13428-019-01243-z

Butman, J., Allegri, R., Harris, P., \& Drake, M. (2000). Fluencia verbal en español. Datos normativos en Argentina. Medicina, 60, 561-564.

Chaigneau, S., Canessa, E., Barra, C., \& Lagos, R. (2018). The role of variability in the property listing task. Behavior Research Methods, 50(3), 972-988. https://doi.org/10.3758/s13428-0170920-8

De Deyne, S., Verheyen, S., Ameel, E., Vanpaemel, W., Dry, M. J., Voorspoels, W., \& Storms, G. (2008). Exemplar by feature applicability matrices and other Dutch normative data for semantic concepts. Behavior Research Methods, 40, 1030-1048.

Devereux, B.J., Tyler, L.K., Geertzen, J., \& Randall, B. (2014). The Centre for Speech, Language and the Brain concept property norms. Behavior Research Methods, 46, 1119-1127.

Fernández Ballesteros, R., Zamarrón, M., Calero, M., \& Tárraga, L. (2007). Cognitive plasticity and cognitive impairment. En R. Fernández Ballesteros 
(Ed.), GeroPsychology. European perspective for an aging world (pp. 145-164). Göttingen: Högrefe \& Huger.

García, A., Ison, M. y Vivas, J. (2019). Conceptual flexibility in schoolchildren: switching between taxonomic and thematic relations. Cognitive Development (52, October-December 2019, 100827). https://doi.org/10.1016/j. cogdev.2019.100827

Garrard, P., Lambon Ralph, M., Hodges, J., \& Patterson, K. (2001). Prototypicality, distinctiveness, and intercorrelation: Analyses of the semantic attributes of living and nonliving concepts. Cognitive Neuropsychology, 18, 125-174.

Gonnerman, L., Andersen, E., Devlin, J., Kempler, D., \& Seidenberg, M. (1997). Double dissociation of semantic categories in Alzheimer's disease. Brain and Language, 57, 254-279.

Hollingshead, A.B. (2011) Four Factor Index of Social Status. Yale Journal of Sociology, 8, 2-52.

Johnson, S. C. (1967). Hierarchical clustering schemes. Psychometrika, 32, 241-254.

Kemper S., \& Sumner, A. 2001. The structure of verbal abilities in young an older Adults. Psychology and Aging, 16(2), 312-322. https://doi. org/10.1037/0882-7974.16.2.312

Kintsch, W. (2001). Predication. Cognitive Science, 25, 173-202.

Kintz, S., \& Wright, H. H. (2017). Semantic knowledge use in discourse: Influence of age. Discourse Processes, 54(8), 670-681.

Kremer, G., \& Baroni, M. (2011). A set of semantic norms for German and Italian. Behavior Research Methods, 43(1), 97-109.

Krzemien, D., \& Richard's, M. (2011). Sabiduría en adultos mayores. Un estudio comparativo de dos modelos. En M C. Richaud \& V. Lemos (Comps.). Psicología y otras ciencias del comportamiento (Vol. I, pp. 173-196). Buenos Aires: ciipme-Conicet.

Lanting, S., Haugrud, N., \& Crossley, M. (2009). The effect of age and sex on clustering and switching during speeded verbal fluency tasks. Journal of the International Neuropsychological Society, 15, 196-204.

Lenci, A., Baroni, M., Cazzolli, G., \& Marotta, G. (2013). blind: a set of semantic feature norms from the congenitally blind. Behavior Research Methods 45(4):1218-1233.

Lindenberger, U., \& Baltes, P. (1997). Intellectual functioning in old and very old age: Cross-sectional results from the Berlin Aging Study. Psychology and Aging, 12(3), 410-432.

Lövden, M., Bäckman, L., Lindenberger, U., Scahefer, S., \& Schiedeck, F. (2010). A theoretical framework for the study of adult cognitive plasticity. Psychological Bulletin, 136(4), 659-676.

MacKay, D. G., \& James, L. E. (2001). The binding problem for syntax, semantics, and prosody: H. M. 'Selective sentences-reading deficits and theoretical syndrome approach. Language and Cognitive Processes, 16(4), 419-460.

Maintenant, C., Blaye, A., \& Paour, J.-L. (2011). Semantic categorical flexibility and aging: Effect of semantic relations on maintenance and switching. Psychology and Aging, 26(2), 461-466.

McRae, K, Cree, G, Seidenberg, M., \& McNorgan, C. (2005). Semantic feature production norms for a large set of living and nonliving things. Behavior Research Methods, 37, 547-559.

Montefinese, M., Buchanan, E., \& Vinson, D. (2018). How well do similarity measures predict priming in abstract and concrete concepts? Recuperado de osf.io/pd3nj

Montefinese, M., Ambrosini, E., Fairfield, B., \& Mammarella, N. (2013). Semantic memory: A feature-based analysis and new norms for Italian. Behavior research methods 45(2), 440-461.

Murphy, G. L. (2002). The big book of concepts. Cambridge, MA: MIT Press.

Navarro, E., \& Calero, M. (2009). Estimation of cognitive plasticity in old adults using dynamic assessment techniques. Journal of Cognitive Education and Psychology, 8, 38-51. 
Pascual, L., Galperín, C., \& Bornstein, M. (1993). La medición del nivel socio-económico y la psicología evolutiva: el caso argentino. RIP, 27(1), 59-74.

Pennequin, V., Fontaine, R., Bonthoux, F., \& Blaye, A. (2006). Categorization deficit in old age: Reality or artefact? Journal of Adult Development, 13(1), 1-9.

Peraita, H., \& Moreno, F. (2006). Análisis de la estructura conceptual de las categorías semánticas naturales y artificiales en pacientes de Alzheimer. Psicothema, 18(3), 492-500.

Peraita, H., Díaz, C., \& Anlló-Vento, L. (2008). Processing of semantic relations in normal aging and Alzheimer's disease. Archives of Clinical Neuropsychology, 23(1), 33-46.

Perea, M. V., Ladera, V., \& Rodríguez, M. A. (2005). Fluencia de acciones en personas mayores. Psicothema, 17(2), 263-266.

Redolar Ripoll, D. (2014). Neurociencia cognitiva. Madrid: Editorial Médica Panamericana.

Riordan, B., \& Jones, M. N. (2010). Redundancy in perceptual and linguistics experience: Comparing feature-based and distributional models of semantic representation. Topics in Cognitive Science, 3(2), 1-43.

Ruts, W., de Deyne, S., Ameel, E., Vanpaemel, W., Verbeemen, T., \& Storms, G. (2004). Dutch norm data for 13 categories and 338 exemplars. Behavior Research Methods, 36(3), 506-515.

Sartori, G., \& Lombardi, L. (2004). Semantic relevance and semantic disorders. Journal of Cognitive Neuroscience, 16, 439-452.

Sautú, R. (1989). Teoría y técnica en la medición del status ocupacional: escalas objetivas de prestigio. Buenos Aires: Instituto Gino Germani, Ciencias Sociales, UBA.

Schaie, K., \& Willis, S. L. (1993). Age difference patterns of psychometric intelligence in adulthood: Generalizability within and across ability domains. Psychology and Aging 8(1), 44-55.

Smith, E. E., Shoben, E. J., \& Rips, L. J. (1974). Structure and process in semantic memory:
Featural model for semantic decisions. Psychological Review, 81, 214-241.

Sommers, M. S. (1996). The structural organization of the mental lexicon and its contribution to age-related declines in spoken-word recognition. Psychology and Aging, 11(2), 333-341.

Taler,V., Lopez Zunini, R., \& Kousaie' S. (2016). Effects of semantic richness on lexical processing in monolinguals and bilinguals. Frontiers in Human Neuroscience. https://doi.org/10.3389/ fnhum.2016.00382

Taylor, K. I., Moss, H. E., \& Tyler, L. K. (2007). The conceptual structure account: A cognitive model of semantic memory and its neural instantiations. En J. Hart y M. Kraut (Eds.), Neural basis of semantic memory (pp. 265-301). Cambridge: Cambridge University Press.

Thornton, R., \& Light, L. (2006). Language comprehension and production in normal aging. En J. E. Birren \& K. W. Schaie (Eds.), Handbook of the psychology of aging (pp. 261-287). Nueva York: Academic Press.

Tyler, L. K., Moss, H. E., Durrant-Peatfield, M., \& Levy, J. P. (2000). Conceptual structure and the structure of concepts: A distributed account of category-specific deficits. Brain \& Language, $75,195-231$.

Véliz, M., Riffo, B., \& Arancibia, B. (2010). Envejecimiento cognitivo y procesamiento del lenguaje: cuestiones relevantes. Revista de Lingüistica Teórica y Aplicada Concepción, 48(1), 75-103. Vinson, D. P., \& Vigliocco, G. (2008). Semantic feature production norms for a large set of objects and events. Behavior Research Methods, 40 (1), 183-190.

Vivas, J., Lizarralde, F., Huapaya, C., Vivas, L., \& Comesaña, A. (2014). Organización reticular de la memoria semántica. Natural Finder y Definition Finder, dos métodos informatizados para recuperar conocimiento. Encontros Bibli 19, 40, 235-252. http://dx.doi. org/10.5007/1518-2924 
Vivas, J., Vivas, L., Comesaña, A., García Coni, A., \& Vorano, A. (2017). Spanish semantic feature production norms for 400 concrete concepts. Behavior Research Methods, 49(3), 1095-1106. https://doi.org/10.3758/s13428-016-0777-2

Vivas, J., Kogan, B., Romanelli, S., Lizarralde, F., \& Corda, L. (2020). A cross-linguistic comparison of Spanish and English semantic norms: Looking at core features. Applied Psycholinguistics, 1-13. https://doi.org/10.1017/S0142716419000523

Vivas, L., Martinez, S. Piccolo, B., García Coni, A., Comesaña, A., \& Vivas, J. (2018). Differences in semantic feature production between healthy older and young adults. Alzheimer's and Dementia, 14(7). https://doi.org/10.1016/j.jalz.2018.06.2525

Vivas, L., Montefinese, M., Bolognesi, M., \& Vivas, J. (2020). Core features: Measures and characterization for different languages. Cognitive Processing. https://doi.org/10.1007/s10339-020-00969-5
Wu, L. L., \& Barsalou, L. W. (2009). Perceptual simulation in conceptual combination: Evidence from property generation. Acta Psychologica, 132, 173-189.

Wulff, D., De Deyne, S., Jones, M., Mata, R., \& The Aging Lexicon Consortium. (2019). New perspectives on the aging lexicon. Trends in Cognitive Sciences. https://doi.org/10.1016/j. tics.2019.05.003

Zannino, G., Perri, R., Pasqualetti, P., di Paola, M., Caltagirone, C., \& Carlesimo, G. (2006). The role of semantic distance in category-specific impairments for living things: Evidence from a case of semantic dementia. Neuropsychologia 44(7), 1017-1028.

Zortea, M., \& Fumagalli de Salles, J. (2012). Estudo comparativo das associações semânticas de palabras entre adultos jovens e idosos. Psicologia: Teoria e Pesquisa, 28(3), 259-266. 